\title{
EVOLUCIÓN DE LAS LÍNEAS DE INVESTIGACIÓN DEL GRUPO PERSPECTIVAS DEL CUIDADO
}

\author{
Ana Julia Carrillo Algarra*, Merideidy Plazas Vargas**, Maida Luz Prieto***
}

\section{Resumen}

Objetivo: describir la trayectoria de la investigación realizada en el grupo Perspectivas del Cuidado de la Facultad de Enfermería de la FUCS. Métodos: estudio descriptivo que incluyó todos los trabajos de investigación realizados de enero 2005 hasta diciembre 2009. Se analizaron la línea, área, tema y metodología. Se encuestó a todos los docentes sobre la motivación de investigar en los temas específicos de su área de conocimiento. Todas las variables fueron analizadas en Stata 10. El trabajo fue aprobado por el Comité de Ética. Resultados: se analizaron 88 trabajos, doce de posgrados. La línea en la que se ha realizado el mayor número es la de cuidado a las personas, destacándose el área de cuidado del adulto $(\mathbf{8 6 / 8 8})$, de los cuales 21 están relacionados con administración de medicamentos. El enfoque que predominó es el cuantitativo. Conclusión: a los docentes les resulta motivante contar con un grupo de investigación estructurado, esto es concordante con el propósito de las filosofías, modelos y teorías de enfermería donde los fenómenos que deben despertar el interés investigativo en sus profesionales son las respuestas que presenta el ser humano.

Palabras clave: investigación, enfermería, metodología, grupo, línea.

\section{THE EVOLUTION OF RESEARCH LINES: FUCS CARE PERSPECTIVES GROUP}

\begin{abstract}
Objective:to describe the evolution of research activity conducted by the Care Perspectives Group of the FUCS Nursing School.Methods: a descriptive clinical trial which included all research projects produced from January 2005 to December 2009 was performed. The particular line, area, topic and methodology were analyzed. Faculty members were surveyed on their motivation to carry on research on specific topics of their special interest profile. An analysis of all variables was performed using Stata 10. This activity was approved by the Ethics Committee. Results: the study included 88 projects, 12 were conducted by postgraduate students. Most of this research was based on human subjects. Adult care was the major topic (86/88), of which 21 projects were related to medication administration. The approach was predominantly quantitative. Conclusions: faculty members were very enthusiastic about counting with a structured research group which agrees with nursing philosophies, models and theories, where the phenomena which must encourage research is human behavior and responses.
\end{abstract}

Key words: research, nursing, methodology, group, line.

Fecha recibido:marzo 13 de 2012 - Fecha aceptado:agosto 3 de 2012

* Enfermera. Profesora Titular, Facultad de Enfermería. de la Fundación Universitaria de Ciencias de la Salud. Bogotá DC. Colombia.
** Especialista en Epidemiología. Profesora Asistente, División de Investigaciones. Fundación Universitaria de Ciencias de la Salud, Bogotá DC, Colombia.

* Enfermera. Instructora Asistente, Facultad de Enfermería. Fundación Universitaria de Ciencias de la Salud, Bogotá DC.Colombia. 


\section{Introducción}

En Colombia las instituciones de educación superior tienen funciones definidas ${ }^{1} \mathrm{y}$ les corresponde transmitir, apropiar, integrar y producir conocimiento que origine cambios en los patrones de conducta en los profesionales en formación y hacerlo extensivo a toda la comunidad, permitiéndoles generar una cultura científica, construir su propia identidad y derivar ventajas comparativas que contribuirán a la validación de sus procesos docentes y sus labores de proyección social. ${ }^{2}$

Se puede entonces inferir que el eje central de estos procesos es la investigación, la cual tiene especial relevancia en la acreditación de los programas de educación superior. ${ }^{1}$ Esto ha permitido que las instituciones se proyecten hacia una investigación sistemática basada en la conformación de grupos y la definición de líneas, en donde se gesta y se produce conocimiento.

En concordancia, la Fundación Universitaria de Ciencias de la Salud (FUCS) contempla en su misión asumir con responsabilidad la formación integral del estudiante en el campo de la ciencia, la investigación y la tecnología, con fundamentos de excelencia científica. ${ }^{3}$ Igual, en sus valores institucionales reconoce el rigor científico como la búsqueda de la excelencia en las actividades y servicios prestados de acuerdo con los estándares nacionales. ${ }^{1,3}$ A su vez, la Fundación impulsa la conformación de grupos y el desarrollo de líneas de investigación en áreas de la salud, ciencias básicas y clínicas, propendiendo fortalecer así los programas de pre y posgrado, la investigación formativa y aplicada, la formación de docentes investigadores y proyectarse a través de la publicación de la producción científica. Como parte de esta cultura investigativa la Facultad de Enfermería durante 2004 implementó una estrategia que conllevó a la definición de dos líneas de investigación: desarrollo histórico y teórico de enfermería y cuidado de las personas, donde se conceptualizaron las áreas que a su vez son alimentadas por diferentes temas y que culminó con la creación del grupo "Perspectivas del cuidado" y su registro en el Departamento Administrativo de Ciencia, Tecnología e InnovaciónCOLCIENCIAS, cuyos resultados fueron publicados en $2009 .{ }^{4}$ Sin embargo, ahora es relevante conside- rar cómo la conformación del grupo ha fortalecido el programa, el cuerpo del conocimiento disciplinar de enfermería, el abordaje de los métodos de investigación que priman en los trabajos adelantados en la facultad, además de identificar las ventajas de tener un grupo con una directriz temática siguiendo los lineamientos del Proyecto Educativo Institucional.

Entonces, el objetivo del presente trabajo fue describir la trayectoria de la investigación en la facultad de enfermería de la FUCS, con base en el análisis de los trabajos realizados por profesores y estudiantes del programa y de las especializaciones de enfermería nefrológica y urológica, en cuidado intensivo y en urgencias, desde el año 2005, fecha de inscripción del grupo en COLCIENCIAS.

Con los resultados se espera brindar herramientas que le permitan a los directivos de la facultad y la FUCS orientar los recursos a las líneas y áreas de mayor producción, generar alianzas estratégicas con grupos y pares académicos, al mismo tiempo fortalecer la divulgación de los productos en revistas indexadas a nivel nacional e internacional. También se espera que los resultados sean de utilidad para las instituciones de educación superior nacionales e internacionales y la generación de conocimiento para la práctica independiente de la profesión de enfermería. ${ }^{5}$

\section{Materiales y métodos}

Se realizó un estudio descriptivo que incluyó todos los trabajos realizados a partir de enero 2005 hasta diciembre 2009. Las investigaciones existentes en la biblioteca de la FUCS procedentes de la facultad de enfermería fueron listadas y se analizó la línea a la que pertenecía el trabajo, área, tema y metodología con base en la información recolectada con el instrumento utilizado para la conformación del grupo. ${ }^{4}$ Se aplicó un instrumento a todos los docentes de las asignaturas de formación profesional a través de una encuesta autodiligenciada, anónima y voluntaria. En esta se exploró sobre la motivación de investigar en su área de conocimiento, los temas específicos, las necesidades y los recursos para el desarrollo de investigaciones 
futuras. Todas las variables fueron analizadas por medio de frecuencias absolutas y relativas en Statal0. El presente trabajo fue aprobado por el Comité de Ética.

\section{Resultados}

Se analizaron 88 trabajos de los cuales 76 corresponden a pregrado. La línea en la que se ha realizado el mayor número de trabajos (86/88) es la de cuidado a las personas, destacándose el área de cuidado del adulto (35/88), de los cuales 21 trabajos se relacionaron con la administración de medicamentos, seguida del área de gestión (Tabla 1).

La mayor parte (59/88) fueron pasantías realizadas en el pregrado. El enfoque cuantitativo predominó (14/88). El tema más frecuente es el de administración de medicamentos, seguido del tema de calidad y del cuidado a los pacientes con alteraciones especificas (Tabla 2). En el momento de la recolección de la información se encontraban vinculados 55 docentes,

\begin{tabular}{|c|c|c|c|}
\hline Líneas/área/tema & Postgrado & Pregrado & Total \\
\hline$n(\%)$ & $12(13.6)$ & $76(86.4)$ & 88 \\
\hline $\mathrm{L}^{*}$. Cuidado a las personas & 11 & 75 & 86 \\
\hline $\mathrm{A}^{\dagger}$. Cuidado madre & 0 & 15 & 15 \\
\hline T‡. Gestación & 0 & 5 & 5 \\
\hline $\mathrm{T}^{\ddagger}$. Pediatría & 0 & 7 & 7 \\
\hline $\mathrm{T}^{\ddagger}$. Rol materno & 0 & 2 & 2 \\
\hline $\mathrm{T \ddagger}$. Puerperio & 0 & 1 & 1 \\
\hline $\mathrm{A}^{\dagger}$. C.Adulto & 3 & 32 & 35 \\
\hline $\mathrm{T} \ddagger$.Admón. medicamentos & 0 & 21 & 21 \\
\hline $\mathrm{T} \ddagger$. Cuidado a los cuidadores & 0 & 1 & 1 \\
\hline $\mathrm{T \ddagger}$. Cuidado a los pacientes $\mathrm{AE}$ & 2 & 7 & 9 \\
\hline $\mathrm{T}$. Cuidado a los pacientes en PD & 0 & 3 & 3 \\
\hline$T^{\ddagger}$. Nutrición & 1 & 0 & 1 \\
\hline$A^{\dagger}$. Gestión & 3 & 19 & 22 \\
\hline $\mathrm{T}^{\ddagger}$. Calidad & 1 & 19 & 20 \\
\hline $\mathrm{T}^{\ddagger}$. Referencia y contrareferencia & 1 & 0 & 1 \\
\hline Tł. Medidas de AS & 1 & 0 & 1 \\
\hline At. Salud mental & 3 & 7 & 10 \\
\hline$T \neq$. Salud mental & 0 & 6 & 6 \\
\hline T‡. Afrontamiento & 3 & 1 & 4 \\
\hline Af. Urgencias y D & 2 & 2 & 4 \\
\hline$T \ddagger$. Emergencias y desastres & 2 & 2 & 4 \\
\hline L*. Fundamentación teórica & 1 & 1 & 2 \\
\hline Af.F. Teóricos & 1 & 1 & 2 \\
\hline \multicolumn{4}{|l|}{ Diseño } \\
\hline Cualitativo & 1 & 9 & 10 \\
\hline Cuantitativo & 8 & 6 & 14 \\
\hline Pasantía & 0 & 54 & 54 \\
\hline Revisión de tema & 0 & 11 & 11 \\
\hline
\end{tabular}

*L: Línea; †A:área de conocimiento; $¥ \mathrm{~T}: T e m a ; A E:$ alteraciones especificas; PD: procedimientos diagnósticos;AS:atención segura;. D: desastres 


\begin{tabular}{|c|c|c|c|c|}
\hline Líneas/área/tema & Cualitativo & Cuantitativo & Pasantia & Revisión de tema \\
\hline$n(\%)$ & $10(11.4)$ & $14(15.9)$ & $59(67.0)$ & $5(5.7)$ \\
\hline L. Cuidado & 8 & 14 & 59 & 5 \\
\hline A. Cuidado madre & 1 & 1 & 12 & 1 \\
\hline T. Gestación & 1 & 0 & 4 & 0 \\
\hline T. Pediatría & 0 & 1 & 5 & 1 \\
\hline T. Rol materno & 0 & 0 & 2 & 0 \\
\hline T. Puerperio & 0 & 0 & $\mathrm{I}$ & 0 \\
\hline A. C. Adulto & 3 & 4 & 27 & 1 \\
\hline T.Admón. medicamentos & 1 & 2 & 18 & 0 \\
\hline T. Cuidado a los cuidadores & 1 & 0 & 0 & 0 \\
\hline T. cuidado a los pacientes $A E$ & 0 & 2 & 6 & 1 \\
\hline T. cuidado a los pacientes en PD & 0 & 0 & 3 & 0 \\
\hline T. Nutrición & 1 & 0 & 0 & 0 \\
\hline A. Gestión & 4 & 6 & 11 & 1 \\
\hline T. Calidad & 4 & 4 & 11 & 1 \\
\hline T. Referencia y contrareferencia & 0 & 1 & 0 & 0 \\
\hline T. Medidas de AS & 0 & 1 & 0 & 0 \\
\hline A. Salud mental & 0 & 2 & 7 & 1 \\
\hline T.Afrontamiento & 0 & 2 & I & 1 \\
\hline A. Urgencias y D & 0 & 1 & 2 & 1 \\
\hline T. Emergencias y desastres & 0 & 1 & 2 & 1 \\
\hline L .Fundamentación teórica & 2 & 0 & 0 & 0 \\
\hline A.F.Teóricos & 2 & 0 & 0 & 0 \\
\hline
\end{tabular}

*L: Línea; **A: área. ${ }^{* * * T: ~ t e m a ; A E: ~ a l t e r a c i o n e s ~ e s p e c i f i c a s ; ~ P D: ~ p r o c e d i m i e n t o s ~ d i a g n o ́ s t i c o s ; ~ A S: ~ a t e n c i o ́ n ~ s e g u r a ; ~ D: ~ d e s a s t r e s . ~}$

de ellos treinta y seis contestaron la encuesta; $94.4 \%$ desean investigar o continuar haciéndolo y $83.3 \%$ manifestaron conocer las áreas y líneas de investigación de la facultad. El área del conocimiento de enfermería que prima para investigar se relaciona con cuidado al adulto, seguido por cuidado a la madre y el niño (Figura 1).

El vacío de conocimiento propio de los docentes estaba conexo con la apropiación del método. Los temas específicos y la formación académica fueron carencias que los encuestados reportaron con más frecuencia (Figura 2). Dentro de los aspectos facilitadores del proceso de investigación se destacaron la motivación y la estructura del grupo. Los recursos que se deberían fortalecer eran la disponibilidad de tiempo y el apoyo económico (Figura 3). Los docentes recomendaron mejorar los incentivos económicos y asignar tiempo para realizar investigación (Figura 4).
El total de docentes que al momento de la recolección de datos no tenía registrada su hoja de vida en CvLac fueron $21(58.3 \%)$ y adujeron dificultad en el acceso a la plataforma ScienTI o déficit en las habilidades propias para diligenciarlo (Figura 5).

\section{Discusión}

La mayoría de los trabajos de investigación se encontraron en la línea de cuidado a las personas, resultado coherente con lo reportado por Murrain en 2009, cuando afirma que el $99 \%$ de los trabajos desarrollados en las especializaciones de la facultad de enfermería abordan el adulto como sujeto de cuidado desde 1999, consistente con la orientación de las mismas hacia estos sujetos de atención ${ }^{6}$ y concordante con los procesos simbióticos de investigación-enseñanza de la facultad, qué apuntan a un egresado preparado para participar en forma activa en la solución de problemas reales 


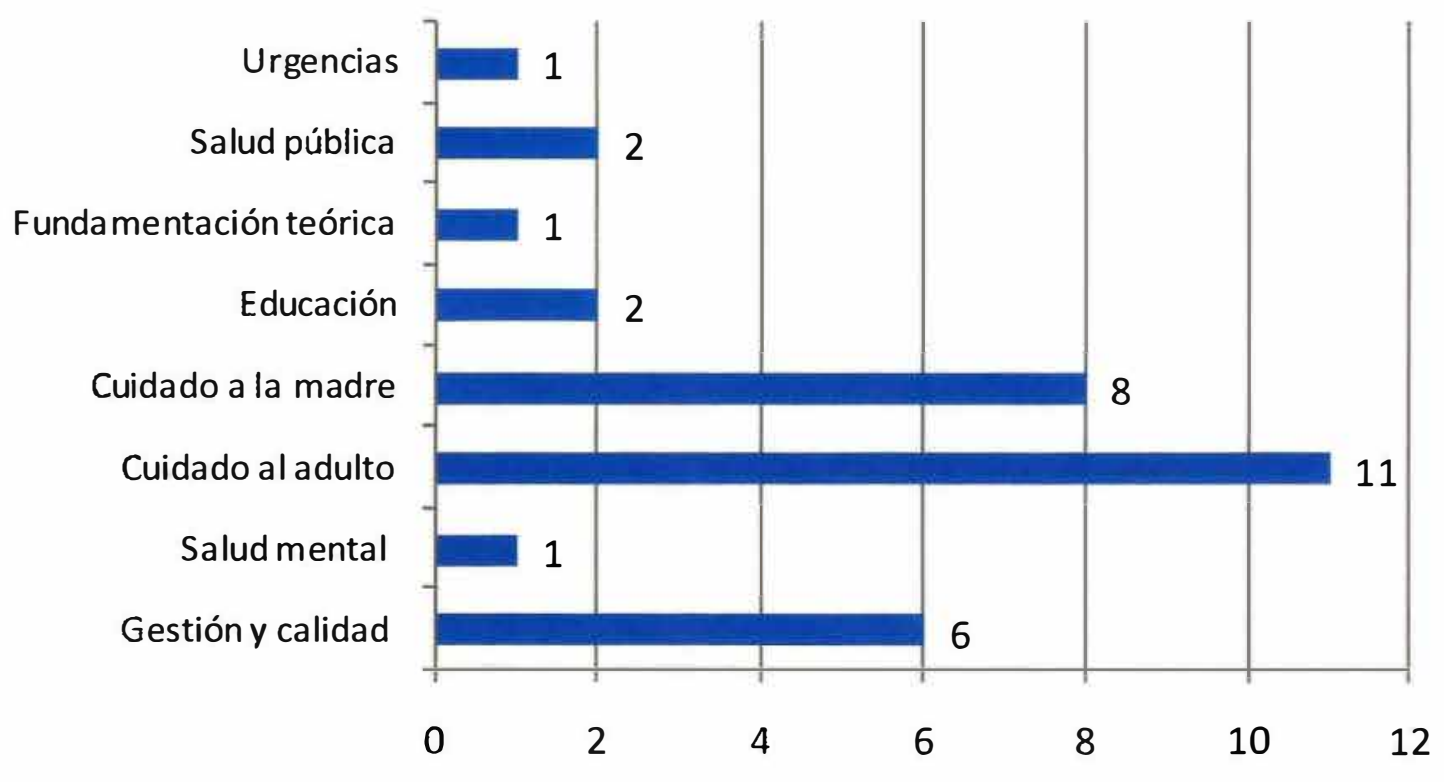

Figura 1. Áreas de interés de los docentes para investigar.

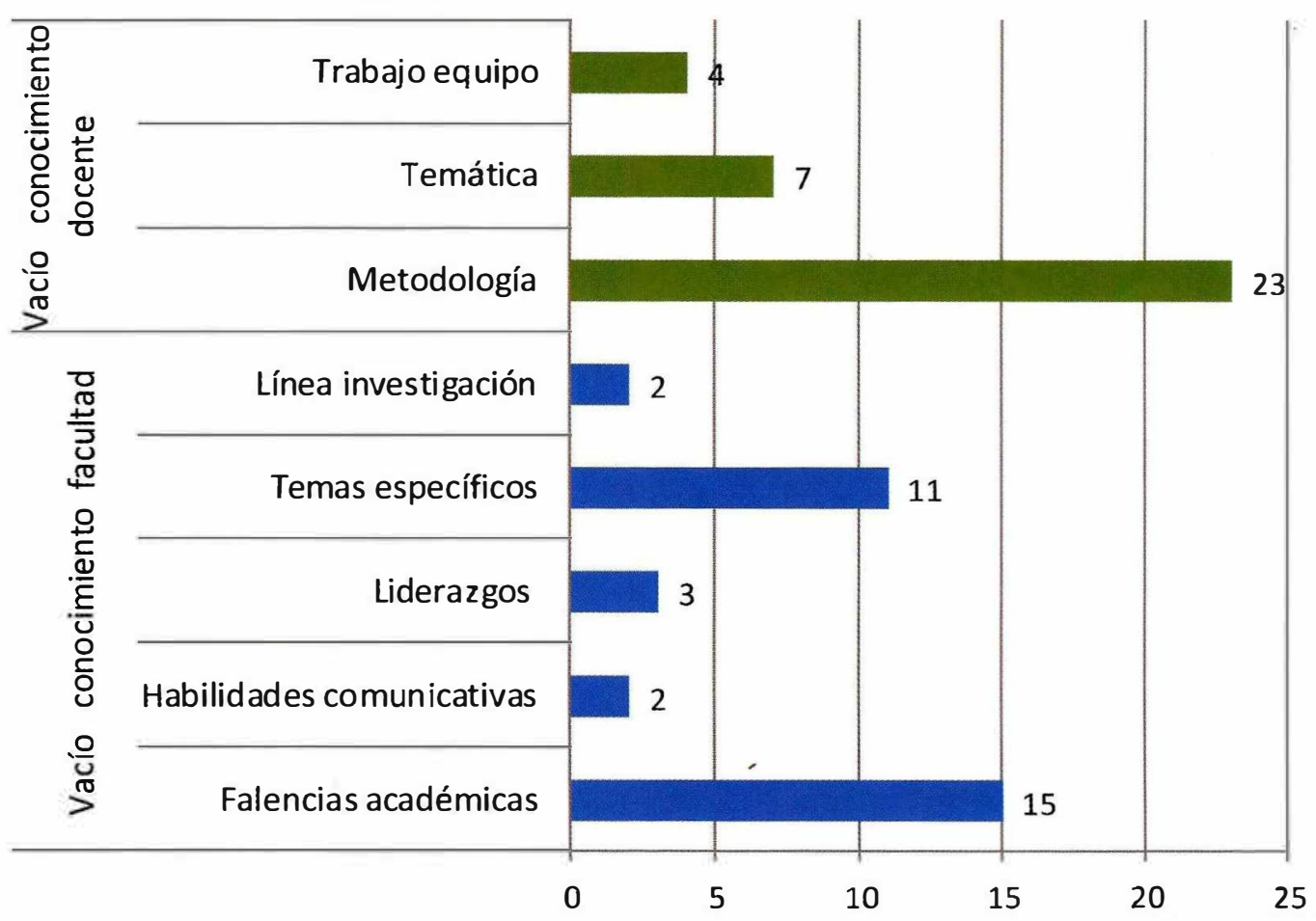

Figura 2. Vacíos percibidos por los docentes. 


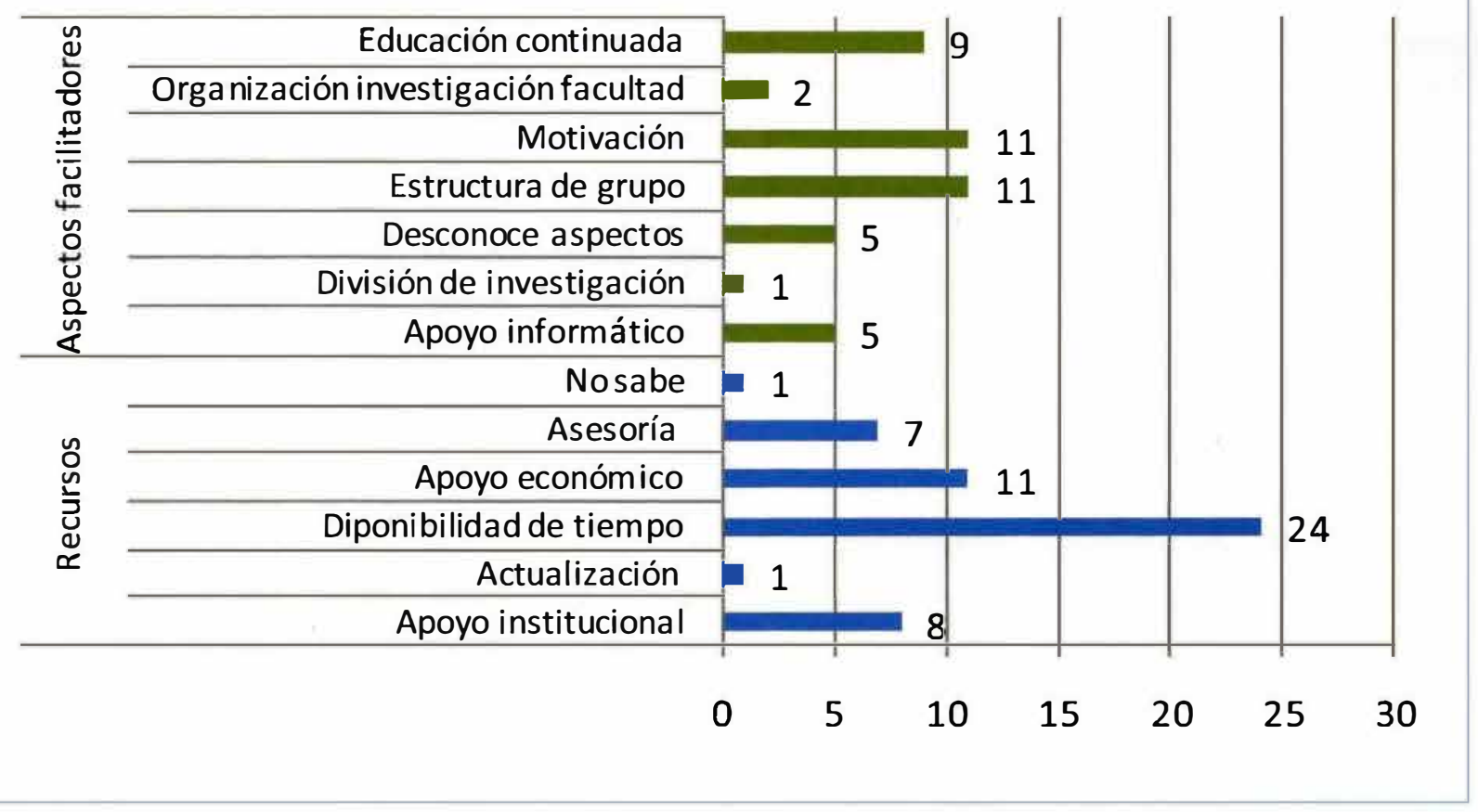

Figura 3. Recursos y aspectos facilitadores.

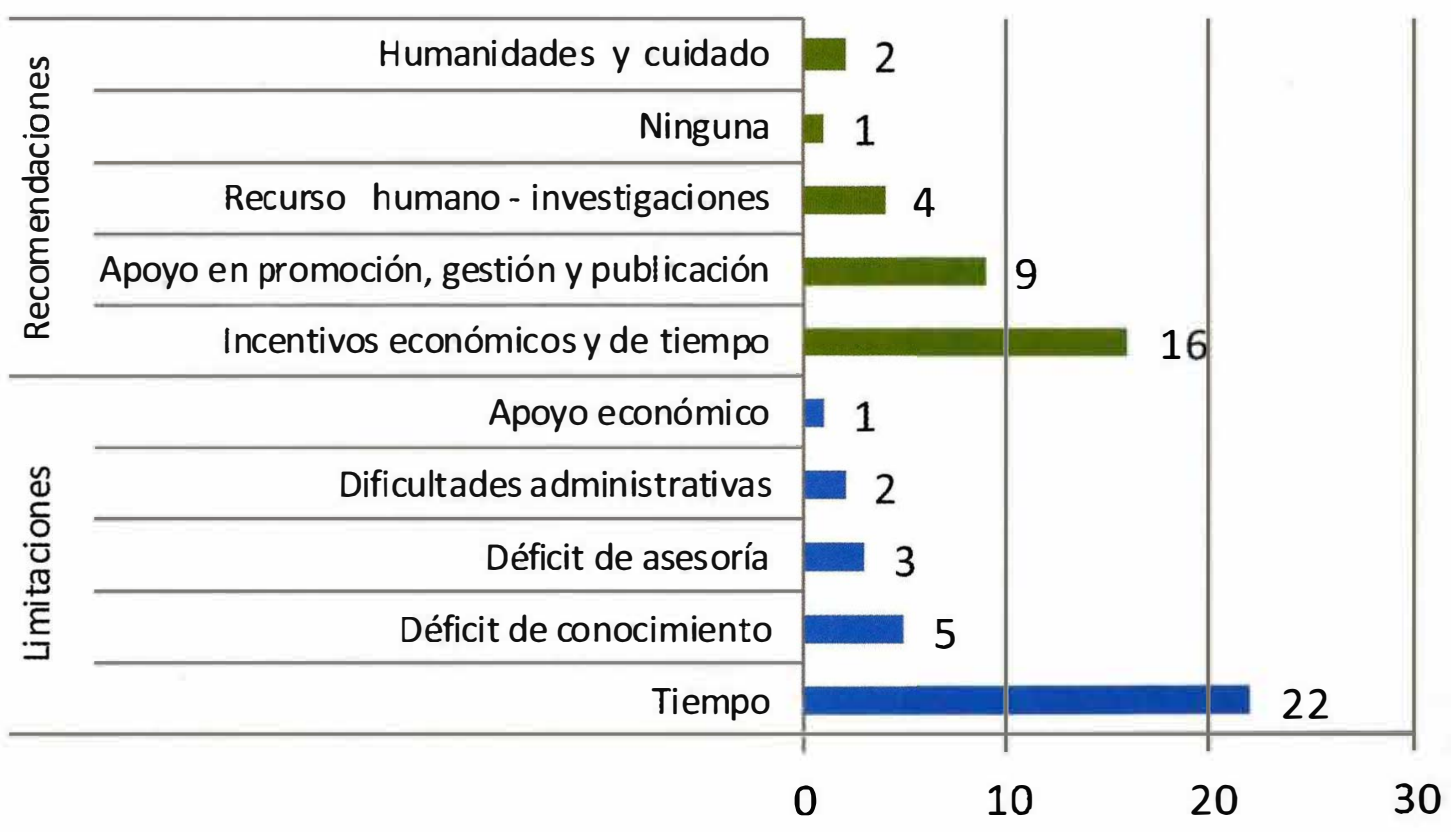

Figura 4. Recomendaciones y limitaciones. 


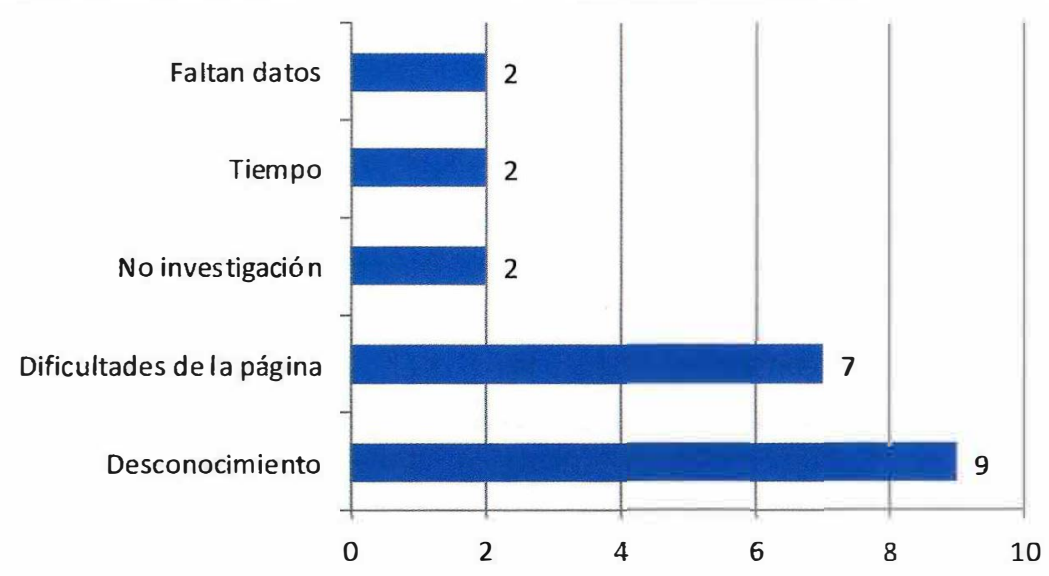

Figura 5. Razones de no registro a CvLac

de salud con un alto sentido ético, humanístico, con énfasis clínico enmarcado en los valores individuales y colectivos que le inspiren un profundo respeto por la dignidad humana ${ }^{7}$

El 23.9\% de los trabajos del área de cuidado al adulto se enfocaron en la administración de medicamentos, tema de especial importancia en el ejercicio de la enfermería, expuesto desde las leyes 911 de 2004 y 266 de 1996 que hacen referencia específica a los deberes del profesional de enfermería frente a la prescripción de medicamentos 8,9 , hasta el desarrollo de investigación relacionada con la solución de problemas de la práctica ${ }^{10}$ y pretendió dar respuesta a la preocupación expuesta por María Elisa Moreno en 2006 en la introducción del módulo, modelos y teorías. donde planteó la preocupación sobre los avances del conocimiento que no se ven reflejados en la práctica que constituye el mundo real de enfermería. ${ }^{11,12}$

El $61.4 \%$ de los trabajos fueron pasantías, producto de la estrategia de flexibilización del currículo implementada por el programa, la cual hace parte de las alternativas de trabajo de grado de los estudiantes de pregrado que permite profundizar en una área de interés y como uno de los productos de la experiencia es realizar un informe que tiende a considerar lineamientos del método científico, consistente con el proceso de investigación formativa propio del pregrado. ${ }^{1,3} \mathrm{La}$ estrategia ha resultado exitosa porque integra los componentes del currículo, la práctica y la investigación.
Se consideró incluir este tipo de trabajos en el proyecto, porque es parte fundamental de la identificación de vacíos de conocimiento, que a la vez aportan fundamentación teórica, convirtiéndose en semillero de preguntas de investigación que nutren las líneas y áreas del grupo perspectivas del cuidado.

Uno de los aspectos para mejorar reportados por los docentes fue la apropiciación del método cientîfico, lo cual llamó la atención teniendo en cuenta que la FUCS en cumplimiento de su función investigativa cuenta con una división que acompaña todos los proceso investigativos en las diferentes facultades. Este resultado puede relacionarse con la rotación de docentes, la cual está determinada por la necesidad de dividir los estudiantes en grupos pequeños para la ejecución de la práctica, generando un alto número de docentes que se contratan solo para ese período y al no tener continuidad no se comprometen con procesos investigativos que requieren mayor dedicación de tiempo.

Como mejoramiento del programa por sugerencia del grupo perspectivas del cuidado y de los pares académicos que los visitaron en su proceso de acreditación voluntaria, ha asumido dos estrategias: la primera, hacer contrataciones anuales que generen tiempo durante el semestre y en el período intersemestral para la realización de trabajos de investigación. La segunda, dictar talleres de capacitación en metodología de la investigación con acompañamiento permanente de la división de investigaciones para todos los docentes 
que ingresan a la facultad, independiente del tipo de contratación.

La división de investigaciones estableció como estrategia el empoderamiento de los docentes de la facultad hacia el grupo, de forma tal que todos los trabajos de grado sean liderados por los docentes integrantes y que apunten hacia el interés del mismo. Además de incrementar la frecuencia de reuniones grupales con el objeto de fortalecer la discusión temática, metodológica y el análisis de las diferentes ideas de investigación.

Dentro de los aspectos destacados se encontró el incremento de interés de los docentes por investigar y el apoyo proporcionado por la universidad, que se vió reflejado en la emisión del manual de incentivos para los docentes dentro del cual los productos que tienen mayor puntaje son aquellos relacionados con procesos de investigación. La división de investigaciones ante la dificultad planteada por los encuestados para registrar su hoja de vida en CvLac, asignó una persona para que realizara el proceso y en forma simultánea entrenar a los docentes en el mismo.

El 15.9\% de los trabajos de investigación del estudio se enmarcan en el método cuantitativo, cuya posible explicación sea la tendencia cuantitativa del modelo de enseñanza y la habilidad investigativa de los docentes por su perfil clínico, lo que coincide con los trabajos monodisciplinares presentados en los coloquios donde predomina este enfoque metodológico, tanto en Colombia como en el mundo según lo publican Murrain y Orts. ${ }^{6,13}$

Los docentes reconocieron como factor de motivación y una ventaja contar con un grupo de investigación estructurado, esto es concordante con el propósito de las filosofías, modelos y teorías de enfermería donde los fenómenos que deben despertar el interés investigativo en sus profesionales, son las respuestas que presenta el ser humano. ${ }^{14}$

Como expresó la doctora Henderson, la enfermería tiene un área independiente, como profesión pone al servicio de la sociedad la producción y el uso responsable del conocimiento y esto se efectúa investigando. En este sentido, es claro que la convierte en la fuente de la ciencia, es toda actividad humana orientada a descu- brir algo desconocido, utiliza el método científico, su actuar genera un cuerpo de conocimientos tecnológicos, epistemológicos, filosóficos y en el caso específico de enfermería, teoría a nivel del cuidado de la salud que orienta el desarrollo profesional, aparte de centrar el conocimiento propio de enfermería referido a los cuidados de las necesidades básicas del individuo, disponiendo de un mayor conocimiento de los fenómenos con que se encuentra el ejercicio profesional y que trata con independencia de otros profesionales. ${ }^{15}$ La aplicación

del método científico en la práctica asistencial de la enfermera, es el proceso de enfermería. Este método permite a las profesionales prestar cuidados de una forma racional, lógica y sistemática ${ }^{14}$, que al trasladarlos a la investigación, hacen de la práctica clínica el contexto de la enfermería moderna.

\section{Referencias}

1 Colombia. Congreso. Capítulo III. Artículo 9 de la Ley 30 de 1992 Por la cual se organiza el servicio público de la Educación Superior (DiarioOficial No. 40.700 de 29 de diciembre de 1992)

2 Arcila O. Las lineas de investigación como elemento articulador de los procesos académicos en la universidad. Nómadas (Col) .1996; Sep-Oct (5):1-139.

3 Fundación Universitaria de Ciencias de la Salud. Proyecto Educativo Institucional. Bogotá: FUCS; 2008.

4 Carrillo A, Muñoz L, Plazas M. Creación y evalución del grupo de investigación Perspectivas del Cuidado. Definición de lineas de investigación. Repert Med Cir. 2009;18(3):161-5

5 Polit D, Hunter B. Investigación Científica de Ciencias de la Salud. Principios y Métodos. 6a ed. Mexico: McGraw Hill Interamaricana; 1994.

6 Murrain E. Tendencias de la Investigación en Enfermería. Repert Med Cir. 2009; 18(2):90-6.

7 Fundación Universitaria de Ciencias de la Salud. Facultad de Enfermería. Perfil del egresado [monografía en Internet]. Bogotá: Fucs; [2012]. [citado 24 Sep. 2012]. Disponible en: http://www.fucsalud.edu.co/index.php/programas-pregrado-fucs/programas-pregrado-enfermeria/661-perfil-estudiantes-enfermeria-fucsfundacion-universitaria-ciencias-salud.html

8 Colombia. Congreso. Capítulo III. Artículo 3 de la Ley 266 de 1996 Por la cual se reglamenta la profesión de enfermería en Colombia y se dictan otras disposiciones (Diario Oficial No. 42.710, del 5 de febrero de 1996)

9 Colombia. Congreso. Capítulo II. Artículo 3 de la Ley 911 de 2004 por la cual se dictan disposiciones en materia de responsabilidad deontológica para el ejercicio de la profesión de Enfermería en Colombia; se establece el régimen disciplinario correspondiente y se dictan otras disposiciones (Diario Oficial No. 45.693 de 6 de octubre de 2004).

10 Acosta G, Medina E. Líneas de investigación en Enfermería. Rev Cubana Enfermer. 1997; 13(2):103-6.

11 Pinto N. El cuidado como objeto del Conocimiento de Enfermería. Av Enferm. 2002;2(1):43.

12 Moreno Fergusson M. Modelos y Teorías. Directrices para la enseñanza de Enfermería en la Educación Superior. 1 ed. Bogotá: ACOFAEN; 2006.

13 Orts M, Cortés P, Moreno M, Arribas M. Identificación de las prioridades de investigación en enfermería en España: Estudio Delphi. Enfermería Clínica. 1999. Jul 27: 10(1):9-13.

14 Dueñas R. Proceso de atención de enfermería [monografía en Internet]. Madrid: Coloplast; 2000. [citado 24 Sep. 2012]. Disponible en: http://www.terra.es/personal/duenas/pae.htm

15 Duran de Villalobos M. Enfermería, desarrollo técnico e investigativo. Bogotá: Universidad Nacional. Facultad de Enfermería; 1998. 